Kui Song

Li Zhang

Guoqing $\mathrm{Hu}$

LNM, Institute of Mechanics, Chinese Academy of Sciences, Beijing, P. R. China

Received June 15, 2011

Revised September 8, 2011

Accepted October 8, 2011
Research Article

\section{Modeling of droplet traffic in interconnected microfluidic ladder devices}

\begin{abstract}
The problem of controlling the droplet motion in multiphase flows on the microscale has gained increasing attention because the droplet-based microfluidic devices provide great potentials for chemical and biological applications. It is critical to understand the relevant physics on droplet hydrodynamics and thus control the generation, motion, splitting, and coalescence of droplets in complex microfluidic networks. Numerical simulations using the volume of fluid algorithm are conducted to investigate the time-dependent dynamics of droplets in gas-liquid multiphase devices. An analytical model based on the electronic-hydraulic analogy is developed to describe the hydrodynamic behavior of the droplets in interconnected microfluidic ladder devices. It is found that the pressure drop caused by the droplets plays a critical role in the droplet synchronization. A fitted formula for pressure drops in the presence of surfactant is achieved by using numerical simulations. Both the numerical and the theoretical results agree well with the corresponding experimental results.
\end{abstract}

Keywords:

Droplet / Microfluidics / Modeling / Synchronization / Volume of fluid

DOI 10.1002/elps.201100320

\section{Introduction}

Since most of the studies of compartmental chemistry and interfacial phenomena and their applications involve twophase flows, recently a surge of interest has arisen in twophase microfluidics, namely as digital microfluidics or droplet-based microfluidics [1-4]. Both droplets and bubbles (hereafter referred to as droplets) in microchannels offer useful tools that have been applied in many fields, for example, chemical kinetics studies [5, 6], biological analysis $[7,8]$, material synthesis $[9,10]$, and even fluidic information-related functions $[11,12]$. Propelled by the necessity to accommodate the increasing requirement for parallel processes, one of the key conditions for the successful and reliable implement of these droplet-based applications is to control droplet traffic in the microfluidic network. Compared with single-phase flow systems, manipulating fluids in such confined multiphase systems containing droplets is much more complicated. Either passive techniques based on hydrodynamic force or capillarity alone [11-18], or active techniques such as electric, dielectric, or pneumatic forces [19-21] can be utilized in the miniaturized devices to successfully regulate the droplet behavior in microchannels.

Correspondence: Professor Guoqing Hu, Institute of Mechanics, Chinese Academy of Sciences, 15 Bei-Si-Huan-Xi-Lu, Beijing 100190, P. R. China

E-mail: guoqing.hu@imech.ac.cn

Fax: +86-10-82543977

Abbreviation: VOF, volume of fluid
One recently developed strategy toward the manipulating of many droplets is self-regulated microchannel networks in which the route and motion of individual droplets are controlled by a well-designed channel layout, rather than by external actuating forces, to form an autonomous behavior of the droplets [11, 12, 22-24]. However, the presence of junctions, bypasses, and often loops in the microchannel network notably increases the complexity of the fluid behavior of droplets. For example, a droplet in a branch channel increases the hydrodynamic resistance of this channel by a given amount, resulting in the change of flow rate in the channel, and it possibly changes the flow lines in all other channels within the network. As a consequence, the route or motion of other droplets will be altered $[11,12,25]$, which again in turn affects the hydrodynamic behavior of existing and subsequent droplets. Such timedependent global coupling of the droplet dynamics makes it difficult to control the droplet traffic and find robust implement of given tasks. It is necessary to develop efficient models to describe and predict the droplet traffic, especially for the large complex microfluidic networks.

Several theoretical works focused on the self-regulated behavior of droplets inside microchannel networks. Jousse et al. [26] derived a compact model of multiphase liquidliquid flow in fluidic networks, based on the well-known "electrical analogy" between laminar flows and electric currents. They simplify the system by treating the fluid resistance within a microchannel as a unique function of the volume fraction of the various phases. Schindler and

Colour Online: See the article online to view Figs. 2-7 in colour. 
Ajdari [27] proposed a simple model for finding robust dynamical behavior and to quantify its response to the changes in the flow conditions and the geometrical parameters of the microchannels. They used this model to analyze the traffic of droplets in "dual microfluidic" networks and found qualitative agreement with the experimental results [11, 12]. Later, Belloul et al. [28] studied the competition mechanism between local collision for small droplets and collective hydrodynamic feedback for large ones. Sessoms et al. [29] developed a phenomenological model to measure the excess hydrodynamic resistance of a channel filled with droplet, by introducing two phenomenological parameters: The hydrodynamic resistance length and the nondimensional droplet mobility coefficient.

In the present study, we focus on the traffic of droplets, without splitting or merging, in the microfluidic networks. A 2-D numerical simulation based on the Volume of Fluid (VOF) scheme is carried out to study the transient motion of bubbles. A theoretical model called "equivalent model" is then proposed to investigate the transit droplet dynamics in a fast way. We explain the principle and advantages of this model, using an interconnected microfluidic ladder device as an example of its application. The effect of surfactant on the pressure drop over a droplet is also considered in the model by least-square fitting of pressure drop to capillary number based on the numerical simulations. It is found that both numerical simulations and analytical analysis agree well with the corresponding experimental results.

\section{Numerical method and theoretical model}

\subsection{Numerical method}

In order to simulate the droplet traffic in the microfluidic network during the time evolution, we choose the VOF method as the numerical scheme [30-32]. We assume the two fluids (gas and liquid) to be Newtonian and incompressible, with uniform surface tension. In the VOF technique, a single set of momentum equations is shared by both fluid phases, and the interface between phases is tracked for each mesh cell throughout the domain by computing the volume fraction $f$ of the gas phase

$f(\vec{x}, t)=\left\{\begin{array}{l}0(\text { a liquid cell }) \\ 1(\text { an gas cell }) \\ 0 \sim 1(\text { an gas }- \text { liquid cell with interface })\end{array}\right.$

The volume fraction function $f$ is governed by the transport equation

$\frac{\partial f}{\partial t}+\nabla \cdot(\vec{v} f)=0$

where $\vec{v}$ is the velocity vector, and $t$ is the time. Then the twophase fluid flow in the microchannel is modeled with the
Navier-Stokes equations

$\nabla \cdot \vec{v}=0$

$\rho \frac{\partial(\vec{v})}{\partial t}+\rho \nabla \cdot(\vec{v} \vec{v})=-\nabla p+\nabla \cdot\left[\mu\left(\nabla \vec{v}+\nabla \vec{v}^{\mathrm{T}}\right)\right]+\rho \vec{g}+\vec{F}$

where $p$ is the static pressure, $\vec{g}$ is the gravitational acceleration, and $\vec{F}$ is a momentum source term related to surface tension. The volume averaged density $\rho$ and dynamic viscosity $\mu$ in each computational cell are calculated based on the volume fraction of one of the phases, $f$

$$
\begin{aligned}
& \rho=\rho_{1} f+\rho_{2}(1-f) \\
& \mu=\mu_{1} f+\mu_{2}(1-f)
\end{aligned}
$$

where indices 1 and 2 represent gas and water in the present simulation, respectively. The gravity term $\rho \vec{g}$ is ignored in the present simulation since such effect is usually insignificant in microfluidic flows.

The Navier-Stokes equations are discretized using a second-order upwind finite-volume scheme. In the incompressible flow, the pressure field is not known a priori and must be obtained as a part of solution. A pressure-velocity coupling algorithm, SIMPLEC (Semi-IMplicit Pressurelinked Equation Consistent) algorithm, along with an algebraic multigrid solver (AMG), is used to iteratively solve the momentum equation and the continuity equation. For the present transient simulations, the time is advanced by the first-order Euler method. A second-order upwind scheme with the Piecewise Linear Interface Construction (PLIC) Method is used to geometrically reconstruct the interface [33]. The source term $\vec{F}$ is the surface tension force per unit volume, which is introduced in the momentum equation by the continuum surface force (CSF) model [34] as

$\vec{F}=\sigma \kappa \vec{n} \delta_{\mathrm{S}}$

where $\sigma$ is the surface tension coefficient, $\kappa$ is the mean curvature of the interfaces, $\vec{n}$ is the unit normal vector of interface, and $\delta_{\mathrm{S}}$ is the interface delta function.

A fully developed laminar parabolic velocity condition is imposed at the inlet of two branches, whereas an outflow boundary condition is imposed at each outlet and a nonslip velocity boundary condition is imposed at the channel walls.

\subsection{Theoretical model}

We here consider the traffic of droplets only in "dual networks," i.e. networks containing transport channels and bypass channels that cannot be accessed by droplets, as shown in Fig. 1. Such dual networks with bypass channels have been used to realize synchronization of droplets motions for the time restoration function in bubble logic systems [12]. A simple model developed in [27] can qualitatively explain this catch-up behavior of droplets in the parallel microchannel. In the following, we analyze the traffic of droplets in the dual microfluidic network based on an algorithm that we call "equivalent model." 


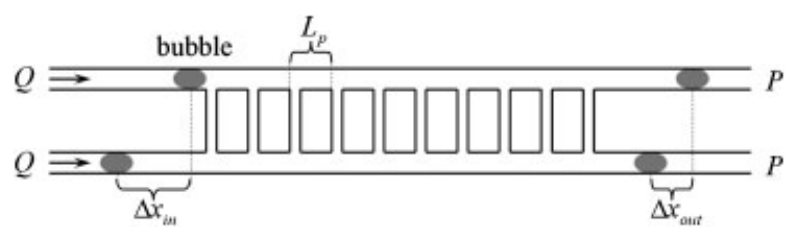

Figure 1. A microfluidic ladder device for droplet synchronization (similar to that used in [12]). $\Delta x_{\text {in }}$ and $\Delta x_{\text {out }}$ are the initial distance and final distance before and after the bypasses between two bubbles, respectively. The bypass channels are inaccessible to bubbles due to their smaller size than droplets.

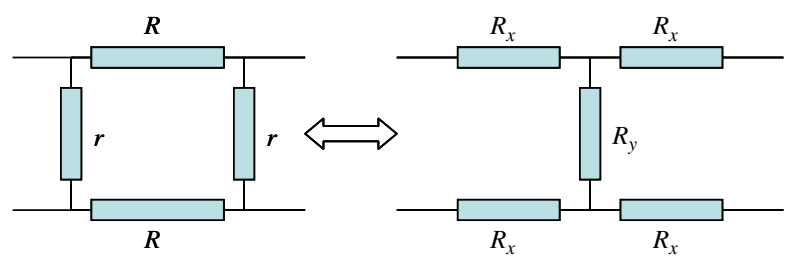

Figure 2. Schematic diagram of principle of the equivalent model.

The laminar flow within in microchannel $i$ can be described by the relationship between pressure drop $\Delta P_{i}$ and flow rate $Q_{i}$

$\Delta P=P_{\text {in }}-P_{\text {out }}=R_{i} Q_{i}$

where $R_{i}$ is the hydrodynamic resistance of microchannel $i$, analogous to the resistance of a conductor in an electric circuit.

The principle of the equivalent method is shown in Fig. 2: The microfluidic structure (A) with two bypass channels can be reconfigured to an equivalent structure (B) with only one bypass channel. The flow resistances of the new structure are linked to the original resistances as

$R_{x}=R r /[2(R+r)], \quad R_{y}=r^{2} /[2(R+r)]$

Following the same principle, a network with $2^{n}(n \geq 1)$ bypasses can be finally simplified to a new equivalent structure with only one bypass. Figure 3 shows the equivalent processing of a network with four bypass channels. We can get the new flow resistances for each level of the equivalent structures as follows:

$$
\begin{aligned}
& n=1: R_{x 1}=R_{1}=R_{0} r_{0} /\left[2\left(R_{0}+r_{0}\right)\right], R_{\gamma 1}=r_{1}=r_{0}^{2} /\left[2\left(R_{0}+r_{0}\right)\right] \\
& n=2:\left\{\begin{array}{l}
R_{x 2}=R_{1}+R_{2}=R_{x 1}+\left(2 R_{1}+R_{0}\right) r_{1} /\left[2\left(2 R_{1}+R_{0}+r_{1}\right)\right] \\
=R_{x 1}+\left(2 R_{x 1}+R_{0}\right) R_{y 1} /\left[2\left(2 R_{x 1}+R_{0}+R_{y 1}\right)\right], \\
R_{y 2}=r_{2}=r_{1}^{2} /\left[2\left(2 R_{1}+R_{0}+r_{1}\right)\right]=R_{y 1}^{2} /\left[2\left(2 R_{x 1}+R_{0}+R_{\gamma 1}\right)\right]
\end{array}\right. \\
& n=3:\left\{\begin{array}{l}
R_{x 3}=R_{x 2}+R_{3}=R_{x 2}+\left(2 R_{x 2}+R_{0}\right) R_{y 2} /\left[2\left(2 R_{x 2}+R_{0}+R_{y 2}\right)\right], \\
R_{y 3}=r_{3}=R_{y 2}^{2} /\left[2\left(2 R_{x 2}+R_{0}+R_{y 2}\right)\right]
\end{array}\right. \\
& \ldots, \text { and } \\
& \left\{\begin{array}{l}
R_{x n}=R_{x(n-1)}+\left(2 R_{x(n-1)}+R_{0}\right) R_{\gamma(n-1)} /\left[2\left(2 R_{x(n-1)}+R_{0}+R_{\gamma(n-1)}\right)\right], \\
R_{y n}=R_{\gamma(n-1)}^{2} /\left[2\left(2 R_{x(n-1)}+R_{0}+R_{\gamma(n-1)}\right)\right]
\end{array}\right.
\end{aligned}
$$

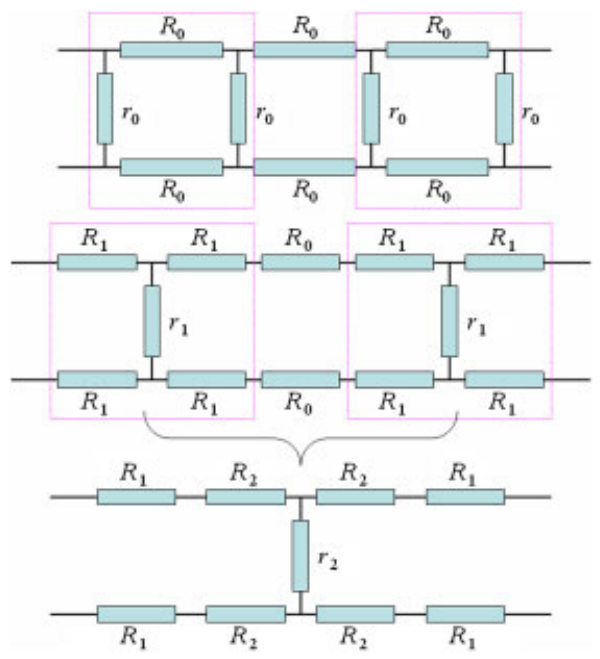

Figure 3. The cascade processing of a ladder device with four bypass channels to an equivalent structure with only one bypass channel.

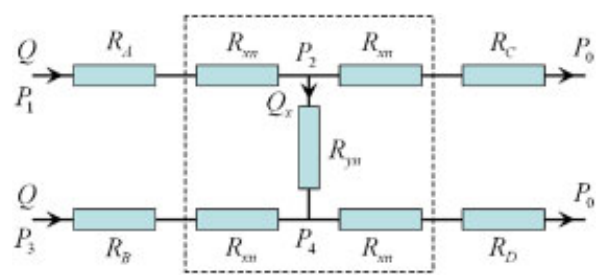

Figure 4. The new microfluidic structure with the part surrounded by dashed lines as the equivalent structure derived from the original $2^{n}$ bypasses. The pressures, flow rates, and resistances are shown in the circuit.

Therefore, many bypass channels can be reduced to one bypass channel, which makes it much easier to decide droplet positions and analyze the droplet traffic. Figure 4 shows the new microfluidic structure with the part surrounded by dashed lines as the equivalent structure derived from the original $2^{n}$ bypasses.

At a node of the branches in the microchannel system, the mass conservation law expression here is that the total flow rate flowing in equals to the total flow rate flowing out, similar to Kirchhoff's law in the electric circuit

$\sum_{j} \varepsilon_{j} Q_{j}=0$

where $j$ represents any of a $j$ branch connecting to the node, and $\varepsilon_{j}= \pm 1$ account for the orientation of the flows.

Droplets in the microchannel significantly affect the flow behavior. If the droplets are sufficiently distant from one other, each droplet then adds an additional increment $R_{b}$ to the resistance of the microchannel. The pressure drop $\Delta P_{b}$ caused by one droplet is estimated as [35]

$\Delta P_{b} \approx 9.4 \sigma \mathrm{Ca}^{2 / 3} / d$

where capillary umber $\mathrm{Ca}=\mu u / \sigma$ and $d$ is the width of microchannel. Using the relationship of $R_{b}=\Delta P_{b} / Q$, 

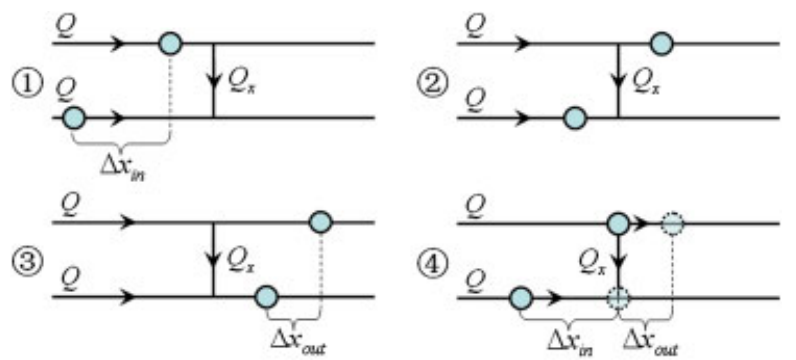

Figure 5. The relative positions of two droplets in the vicinity of the bypass channel.

we obtain

$R_{b}=A Q^{-1 / 3}, A=9.4 \mu^{2 / 3} \sigma^{1 / 3} / d^{5 / 3}$

In the equivalent structure with one bypass channel, there exist only three possible relative positions of two droplets, as shown in Fig. 5 (1), (2) and (3). It is clear that the relative distance between two droplets will decrease only in case (2) in which the flow rate through the bypass is not zero. With such positions of droplets, the total resistance $R_{x n}+R_{C}$ of the upper downstream channel increases to $R_{x n}+R_{C}+R_{b}$, or $R_{x n}+R_{C}+A\left(Q-Q_{x}\right)^{-1 / 3}$, whereas the total resistance $R_{B}+R_{x n}$ of the lower upstream channel increases to $R_{B}+R_{x n}+A Q^{-1 / 3}$ (also see Fig. 4). Using the relationships between Eqs. (6) and (9), we have the following set of equations:

$\left\{\begin{array}{l}P_{1}-P_{2}=\left(R_{A}+R_{x n}\right) Q \\ P_{2}-P_{0}=\left[R_{x n}+R_{C}+A\left(Q-Q_{x}\right)^{-1 / 3}\right]\left(Q-Q_{x}\right) \\ P_{2}-P_{4}=R_{y n} Q_{x} \\ P_{3}-P_{4}=\left(R_{B}+R_{x n}+A Q^{-1 / 3}\right) Q \\ P_{4}-P_{0}=\left(R_{x n}+R_{D}\right)\left(Q+Q_{x}\right)\end{array}\right.$

After simple algebraic manipulations, $Q_{x}$ can be expressed as

$Q_{x}=\frac{A\left(Q-Q_{x}\right)^{-1 / 3} Q}{R_{y n}+2\left(R_{C}+R_{x n}\right)+A\left(Q-Q_{x}\right)^{-1 / 3}}$

Assume the time needed for traveling of droplets in Fig. 5(4) is $t$, the mass conservation gives

$Q t=\Delta x_{i n} d$

$\left(Q-Q_{x}\right) t=\Delta x_{\text {out }} d$

Combining Eqs. (13) and (14) leads to

$A^{3} \eta^{2} /[B(1-\eta)]^{3}-Q=0$

where $\eta=\Delta x_{\text {out }} / \Delta x_{\text {in }}, B=R_{y n}+2\left(R_{C}+R_{x n}\right)$. Equation (15) answers how the two droplets with initial distance $\Delta x_{\text {in }}$ can be synchronized, depending on the geometrical parameters of fluidic network and flow parameters.

If the total number $k$ is not exactly the power of 2 , it is little difficult to directly apply the equivalent analysis. Here, we try to establish a similar equation based on numerical fitting. By introducing $R_{x n}=E R_{0}, R_{\gamma n}=F R_{0}$, and
$B=2 R_{C}+(2 E+F) R_{0}$, Eq. (15) becomes

$\frac{A^{3}}{\left[2 R_{C}+(2 E+F) R_{0}\right]^{3}} \frac{\eta^{2}}{(1-\eta)^{3}}-Q=0$

where $(2 E+F)$ depends on the number of bypasses and the ratio of $r_{0} / R_{0}$. Using Eq. (8), $(2 E+F)$ can be obtained for different bypass number and a given value of $r_{0} / R_{0}$. For the ladder-like microfluidic network used in the experiments of[12], we have $r_{0} / R_{0}=10.67$ and $2 E+F=\mathrm{e}^{1.2382+0.2575 / \ln k}$ for $k \geq 2$.

\section{Results and discussion}

We begin with the VOF simulation of droplet motion in a ladder device used in [12] as a passive bubble synchronizer, as shown in Fig. 1. This planar device consists of a fluidic ladder network with two wide parallel channels $(50 \mu \mathrm{m}$ wide) with equal flow and ten interconnected bypass channels $(25 \mu \mathrm{m}$ wide, $100 \mu \mathrm{m}$ long, and $75 \mu \mathrm{m}$ pitch). The dispersed gas phase is nitrogen $\left(\rho_{1}=1.16 \mathrm{~kg} / \mathrm{m}^{3}\right.$, $\left.\mu_{1}=1.76 \times 10^{-5} \mathrm{Pas}\right)$ and the continuous liquid phase is water $\left(\rho_{2}=998 \mathrm{~kg} / \mathrm{m}^{3}, \quad \mu_{2}=0.001 \mathrm{~Pa} \mathrm{~s}\right)$ with Tween-20 surfactant $(2 \% \mathrm{w} / \mathrm{w})$. Addition of Tween-20 surfactant lowers the interfacial tension $\sigma$ between nitrogen and water to a value of $0.035 \mathrm{~N} / \mathrm{m}$ [15]. The contact angle between nitrogen and channel wall is assumed to be $150^{\circ}$. All the numerical simulations were conducted using a commercial CFD software package (CFD-ACE 2008, ESI). In simulations, a numerical damping parameter is added in CFDACE's VOF module to prevent capillary waves and to match experimental observation of bubble traffics. We believe that the combined effects of the lower surface tension and the numerical damping make the simulations close to the practical situations with added surfactant. Recently, there are a few 3-D simulation studies on droplet dynamics within relatively simple T-conjunction microfluidic configurations [36, 37]; the computational cost of a fully 3-D simulation, however, can be very high especially for the transient process of droplet traffic in the present complex network. The 2-D approximation would be accurate with high aspect ratio microchannels. We assume here that it provides qualitatively correct results for the rectangular microchannels by freezing the degree of freedom in the vertical dimension. Such 2-D approximation was proven effective by the previous studies such as droplet mixing in a drop moving through a serpentine channel [38] and droplet breakup in microfluidic T-junctions [39]. The 2-D computational domain was divided into about 14400 quadrilateral meshes. The grid independency was tested by doubling the number of meshes and no discernible difference was found in simulation results using different meshes. Droplets were initially placed at the channel upstream with a distance apart from each other and the motion was driven by imposing a parabolic inlet velocity at two branch inlets. A fixed time advancing step of $2 \times 10^{-6} \mathrm{~s}$ was used in all computing. 

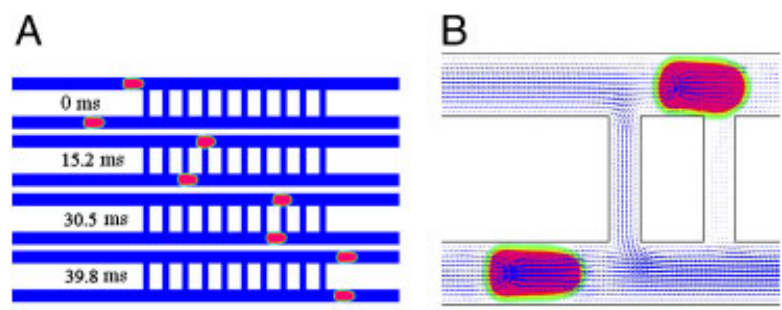

Figure 6. The VOF numerical simulations of the bubble synchronization experiment in [12]. (A) The motion of two bubbles initially $150 \mu \mathrm{m}$ apart under a flow rate of $0.5 \mu \mathrm{L} / \mathrm{s}$; (B) close-up of the flow field showing the flow through the bypass between two bubbles.

Figure 6A shows the synchronization process of two bubbles initially $150 \mu \mathrm{m}$ apart, under a flow rate of $0.5 \mu \mathrm{L} / \mathrm{s}$. The present numerical simulation results agree well with the experimental findings in [12]. When two bubbles are travelling in the parallel microchannels simultaneously, the bypass channels between these two bubbles allow for net flow from the channel with the leading bubble to the one with the lagging bubble. The resultant velocity gradient of the carrying fluid makes two bubbles synchronized. After passing through all the ladder-like bypasses, two bubbles move at almost identical speed. Figure 6B shows the closeup velocity field around two bubbles and bypasses, and the net flow from one transport channel to another via the bypass between two bubbles can be clearly seen.

We also studied the synchronization efficiency of this microfluidic ladder device at different inlet flow velocities. Figure 7 compares the distance $\left(\Delta x_{\text {out }}\right)$ of two bubbles after passing the ladder bypasses at three flow velocities $(0.143$, 0.185 , and $0.24 \mathrm{~m} / \mathrm{s})$. As expected, the slower the velocity, the better synchronization can be achieved. For the $0.24 \mathrm{~m} / \mathrm{s}$ flow velocity, the leading bubble still moves about half of the bubble length ahead after ten bypasses. More or wider bypasses are needed to get better synchronization for high flow rates. However, since here even the slowest velocity $(0.143 \mathrm{~m} / \mathrm{s})$ is already relatively large in the sense of microfluidics, such ladder devices should be able to provide satisfactory synchronization functions for ordinary applications based on droplets or bubbles.

The present equivalent model permits fast evaluation and optimization of droplet-based microfluidic devices. With regard to the ladder devices, we can study the effect of different parameters, such as the number of bypasses, their hydrodynamic resistance, and their pitch distances. Figure 8 compares synchronization efficiency predicted by the equivalent mode and the model of [27]. At this stage, we use $\Delta P_{b} \approx 9.4 \sigma \mathrm{Ca}^{2 / 3} / d$ as the pressure drop caused by a single bubble, without considering the effects of surfactant [35]. We use the ratio of the final distance to the initial distance between two bubbles, $\eta=\Delta x_{\text {out }} / \Delta_{\text {in }}$, to represent the synchronization performance. A small $\eta$ value close to zero means that the lagging bubble catches up the leading one. As shown in Fig. 8, our equivalent model agrees with the model of [27] very well. Both models show that $\eta$ only

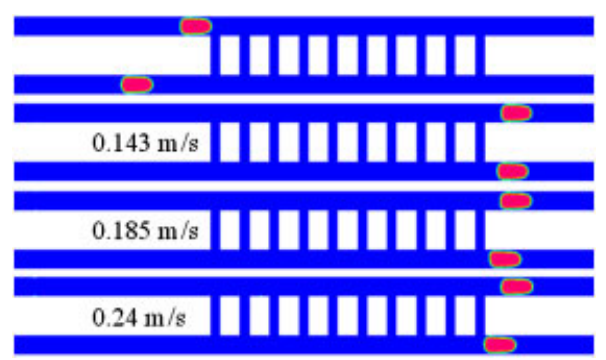

Figure 7. Comparison of synchronization efficiency of the ladder device under different velocities $(0.143,0.185$, and $0.24 \mathrm{~m} / \mathrm{s})$.

slightly changes within a certain range of initial distances and inlet velocities. As shown in Fig. 8C, increasing the number of bypasses from 3 to 16 only weakly decreases the final distance between two bubbles, which was previously pointed out in [27].

Although the equivalent model and the mode of [27] agree well with each other, their predictions significantly deviate from the corresponding experiment and numerical simulation. Both the experiments in [12] and the present VOF numerical simulation clearly show that two bubbles reach at synchronization status after passing ten bypasses. This inconsistency between analytical models and experimental/numerical results arises from the pressure drop caused by bubble. The expression for the pressure drop in Eq. (10) is derived by ignoring the effects of added surfactant. However, surfactant may have strong effects on the pressure drop necessary to push a bubble through a confined channel. One explanation of the bubble resistance increasing due to the added surfactant was made by Probstein [40]. Probstein stated that the surfactant film behaves like an incompressible thin solid membranes at which the liquid velocity drops to zero and the velocity change across the lubrication film is much sharper than in the absence of surfactant. He found that pressure drop with surfactant is about two orders of magnitude higher than it is in the case without surfactant. Recently, Fuerstman et al. [41] conducted experiments to study surfactant's effects on droplet and found that the presence of surfactant can reduce the droplet velocity by up to $50 \%$. In the current microfluidic ladder devices, the hydrodynamic resistance of droplets has the strongest influence on the synchronization. To elucidate the effects of different bubble resistances on the bubble traffic, we numerically simulated the nitrogen-water two-phase flows under various ranges of capillary number $\mathrm{Ca}$. The numerical results were used to obtain a fitted parameter that links the pressure drops of bubble to $\mathrm{Ca}^{2 / 3}$ through the least square fitting. Figure 9 shows the fitting curve of pressure drops over the Ca number from 0.002 to 0.008 . Therefore, the pressure drop caused by a bubble is changed to

$\Delta P_{b} \approx 80 \sigma \mathrm{Ca}^{2 / 3} / d$

which is much higher than the value estimated by Eq. (10) without considering surfactant effects. It is noted that the results presented here are only a rough estimate for the effects of surfactant on the bubble resistance, by lowering 

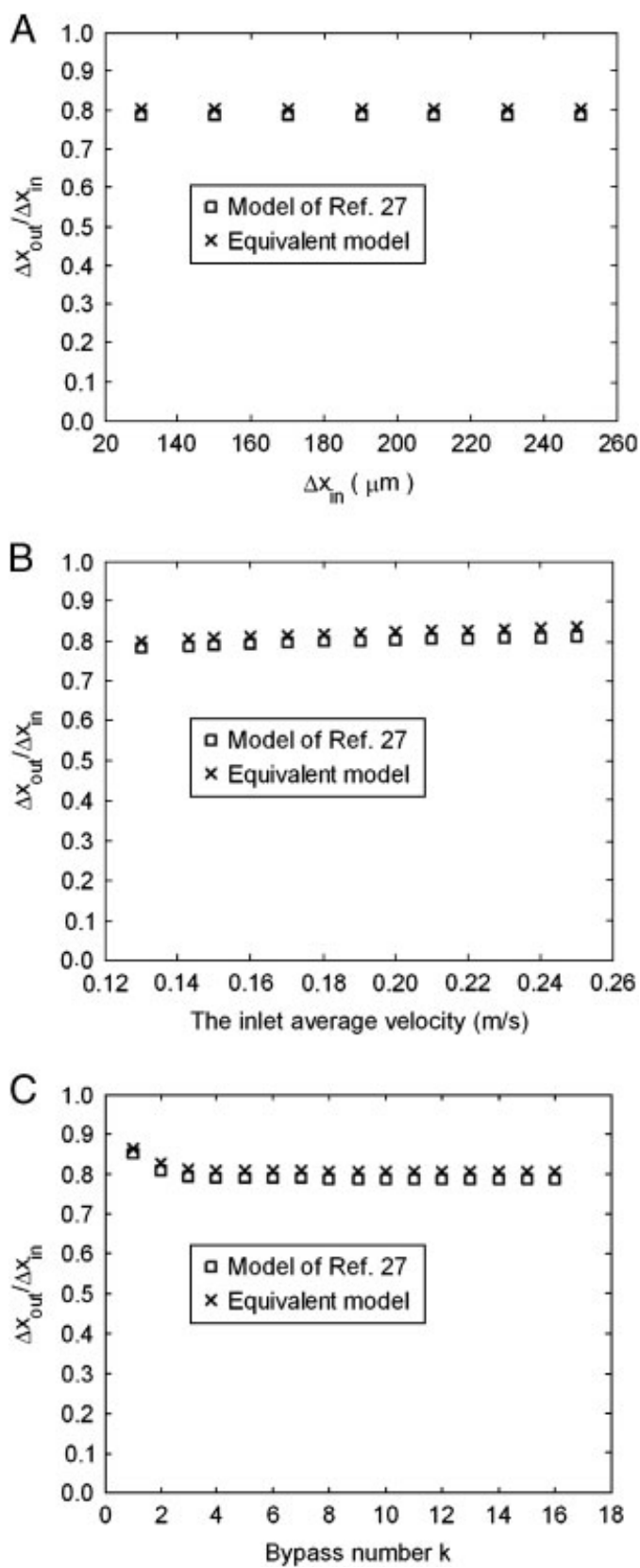

Figure 8. Comparison of the synchronization efficiency predicted using equivalent mode and model of [27]. (A) Effects of initial distance, with inlet velocity of $0.143 \mathrm{~m} / \mathrm{s}$ and ten bypasses; (B) effects of inlet velocity, with initial distance of $150 \mu \mathrm{m}$ and ten bypasses; (C) effects of bypass number, with inlet velocity of $0.143 \mathrm{~m} / \mathrm{s}$ and ten bypasses.

the surface tension in VOF simulations. The pressure drop that we obtained is about ten times higher than it is in the absence of surfactant, whereas the corresponding capillary number changes only about $50 \%$.

Figure 10 shows the predictions by the equivalent model and the model of [27] using the fitted pressure drops. Figure $10 \mathrm{~A}$ plots the synchronization performance $\Delta x_{\text {out }} / \Delta x_{\text {in }}$ obtained by two models, experiments, and numerical

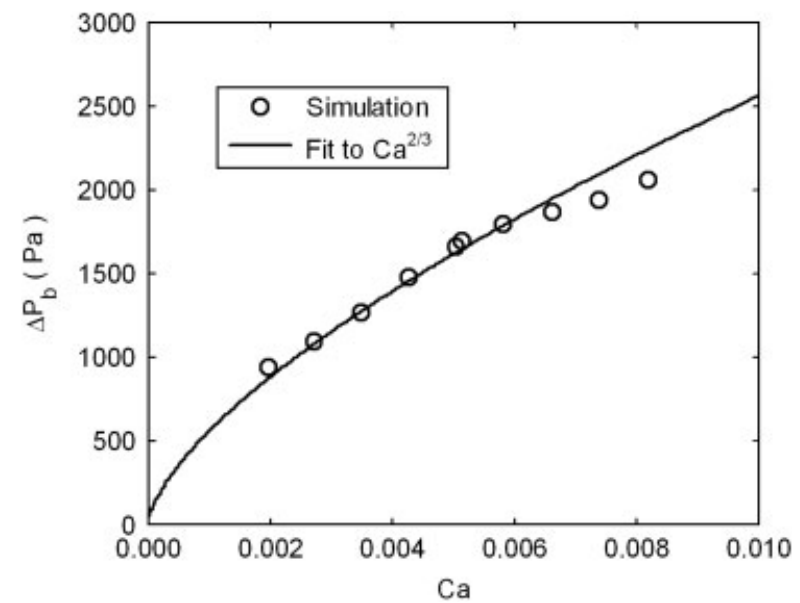

Figure 9. The fitting curve of pressure drops over $\mathrm{Ca}$ number using the simulation results in a surfactant-mediated nitrogenwater multiphase system. The pressure drop caused by a bubble is estimated as $\Delta P_{b} \approx 80 \sigma \mathrm{Ca}^{2 / 3} / d$.
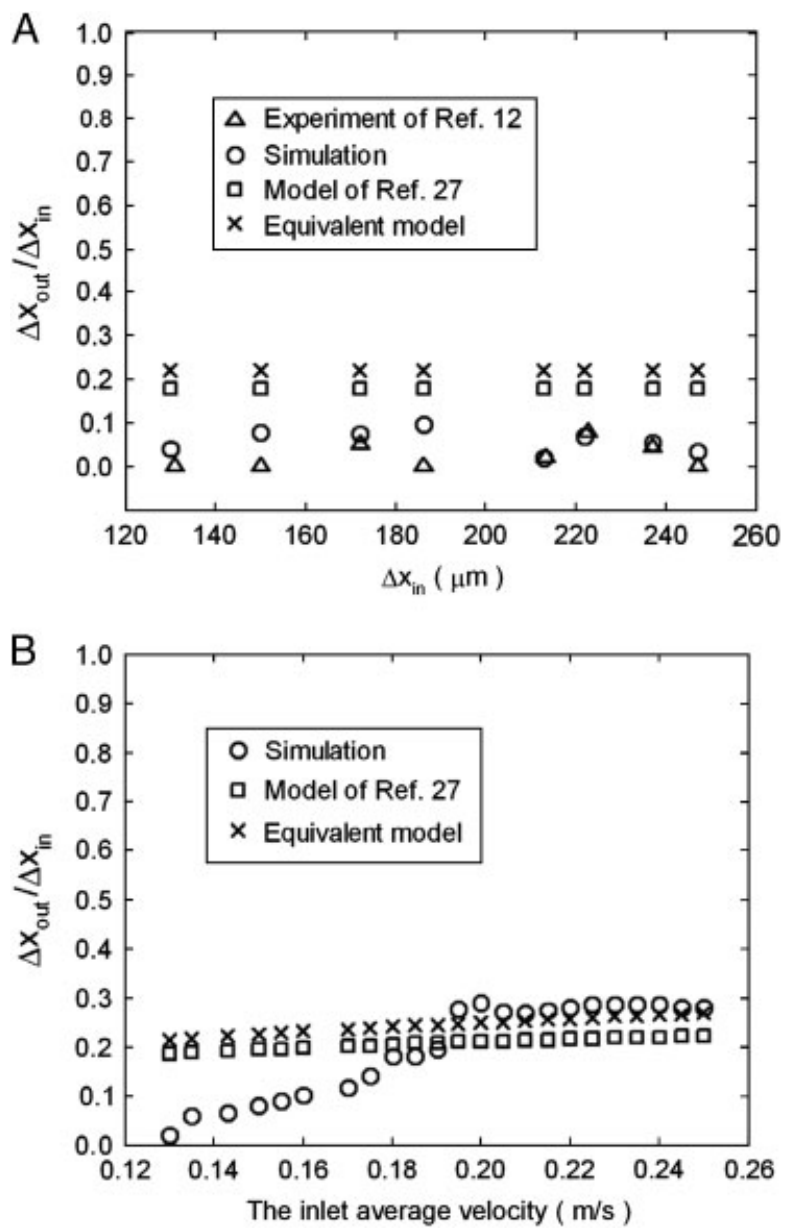

Figure 10. Comparison of the synchronization efficiency predicted using equivalent mode and model of [27] with the fitted bubble pressure drops. (A) Effects of initial distance, with inlet velocity of $0.143 \mathrm{~m} / \mathrm{s}$ and ten bypasses; (B) effects of inlet velocity, with initial distance of $150 \mu \mathrm{m}$ and ten bypasses. 
simulations in the ladder devices with different initial distances $\Delta x_{\text {in }}$ under the same inlet velocity of $0.143 \mathrm{~m} / \mathrm{s}$. By modifying the pressure drops, both models now can repeat the experimental and numerical results much better than before. Due to the lack of experimental data, Fig. 10B compares only the results from two models to the numerical simulations for various inlet velocities. Although there are some differences between numerical simulations and models' predictions at low velocities, overall they agree with one another to a satisfactory extent.

\section{Concluding remarks}

We have developed a theoretical model based on the cascade of equivalent simplification to analyze the traffic of droplets in the microfluidic ladder devices. With this approach, a microfluidic network with many interconnected bypass channels can be reduced to an equivalent structure with only one bypass, which makes it much easier to analyze the traffic of droplets. The derived model can take into account the key parameters of droplet manipulations, such as the geometry of the microfluidic network, the flow rate, and the resistance of droplet. We have performed both VOF numerical simulations and theoretical analysis to capture the bubble synchronization observed in the previous experiments. The dramatic increase of the pressure drop and thus the bubble resistance caused by the presence of surfactant in the multiphase flows is also considered in the model. By fitting the pressure drop in terms of the capillary number, the present model and other existing model have yielded better predictions with regard to the experimental results. This model can be used to evaluate and optimize related droplet-based microfluidic devices as a fast and an efficient tool. For example, we are currently extending this model to study the bistability behavior of droplet flow in other microchannel networks.

This work was supported by the National 973 Project (Grant No. 2011CB707604) and Knowledge Innovation Program of CAS (KJCX2-YW-H18).

The authors have declared no conflict of interest.

\section{References}

[1] Joanicot, M., Ajdari, A., Science 2005, 309, 887-888.

[2] Gûnther, A., Jensen, K. F., Lab Chip 2006, 6, 1487-1503.

[3] Fair, R. B., Microfluid. Nanofluid. 2007, 3, 245-281.

[4] Teh, S., Lin, R., Hung, L., Lee, A. P., Lab Chip 2008, 8, 198-220.

[5] Song, H., Tice, J. D., Ismagilov, R. F., Angew. Chem. Int. Ed. 2003, 42, 767-772.

[6] Song, H., Ismagilov, R. F., J. Am. Chem. Soc. 2003, 125, 14613-14619.
[7] Huebner, A., Olguin, L. F., Bratton, D., Whyte, G., Huck W. T. S., de Mello, A. J., Edel, J. B., Abell, C., Hollfelder, F., Anal. Chem. 2008, 80, 3890-3896.

[8] Shi, W., Wen, H., Lu, Y., Shi, Y., Lin, B., Qin, J., Lab Chip 2010, 10, 2855-2863.

[9] Shestopalov, I., Tice, J. D., Ismagilov, R. F., Lab Chip 2004, 4, 316-321.

[10] Duraiswamy, S., Khan, S. A., Small 2009, 5, 2828-2834.

[11] Fuerstman, M. J., Garstecki, P., Whitesides, G. M., Science 2007, 315, 828-832.

[12] Prakash, M., Gershenfeld, N., Science 2007, 315, 832-835.

[13] Thorsen, T., Roberts, R. W., Arnold, F. H., Quake, S. R., Phys. Rev. Lett. 2001, 86, 4163-4166.

[14] Anna, S. L., Bontoux, N., Stone, H. A., Appl. Phys. Lett. 2003, 82, 364-366.

[15] Garstecki, P., Fuerstman, M. J., Stone, H. A., Whitesides, G. M., Lab Chip 2006, 6, 437-446.

[16] Baroud, C. N., Gallaire, F., Dangla, R., Lab Chip 2010, 10 2032-2045.

[17] Barbier, V., Willaime, H., Tabeling, P., Jousse, F., Phys. Rev. E 2006, 74, 046306.

[18] Zhang, Q., Zeng, S., Qin, J., Lin, B., Electrophoresis 2009, 30, 3181-3188.

[19] Chabert, M., Dorfman, K. D., Viovy, J., Electrophoresis 2005, 26, 3706-3715.

[20] Link, D. R., Grasland-Mongrain, E., Duri, A., Sarrazin, F., Cheng, Z., Cristobal, G., Marquez, M., Weitz, D. A., Angew. Chem. Int. Ed. 2006, 118, 2618-2622.

[21] Kaneda, S., Ono, K., Fukuba, T., Nojima, T., Yamamoto, T., Fujii, T., Electrophoresis 2010, 31, 3719-3726.

[22] Engl, W., Roche, M., Colin, A., Panizza, P., Ajdari, A., Phys. Rev. Lett. 2005, 95, 208304.

[23] Jousse, F., Farr, R., Link, D. R., Fuerstman, M. J., Garstecki, P., Phys. Rev. E 2006, 74, 036311.

[24] Cheow, L. F., Yobas, L., Kwong, D., Appl. Phys. Lett. 2007, 90, 054107.

[25] Cristobal, G., Benoit, J., Joanicot, M., Ajdari, A., Appl. Phys. Lett. 2006, 89, 034104.

[26] Jousse, F., Lian, G., Janes, R., Melrose, J., Lab Chip 2005, 5, 646-656.

[27] Schindler, M., Ajdari, A., Phys. Rev. Lett. 2008, 100, 044501.

[28] Belloul, M., Engl, W., Colin, A., Panizza, P., Ajdari, A., Phys. Rev. Lett. 2009, 102, 194502.

[29] Sessoms, D. A., Belloul, D. A., Engl, W., Roche, M., Courbin, L., Panizza, P., Phys. Rev. E 2009, 80, 016317.

[30] Hirt, C. W., Nichols, B. D., J. Comput. Phys. 1981, 39 201-225.

[31] Harvie, D. J. E., Fletcher, D. F., J. Comput. Phys. 2000, 162, 1-32.

[32] Aulisa, E., Manservisi, S., Scardovelli, R., Zaleski, S., J. Comput. Phys. 2003, 192, 355-364.

[33] Youngs, D. L., Time-Dependent Multi-material Flow with Large Fluid Distortion, Academic Press, New York 1982. 
[34] Brackbill, J. U., Kothe, D. B., Zemach, C., J. Comput. Phys. 1992, 100, 335-354.

[35] Bretherton, F. P., J. Fluid Mech. 1961, 10, 166-188.

[36] De Menech, M., Garstecki, P., Jousse, F., Stone, H. A., J. Fluid Mech. 2008, 595, 141-162.

[37] Sivasmy, J., Wong, T.-N., Nguye, N.-T., Kao, L. T.-H., Microfluid. Nanofluid. 2011. DOI: 10.1007/s10404-0110767-8.

[38] Muradoglu, M., Stone, H. A., Phys. Fluids 2005, 17, 073305.
[39] Jullien, M.-C., Ching, M.-J., Cohen, C., Menetrier, L., Tabeling, P., Phys. Fluids 2009, 21, 072001.

[40] Probstein, R. F., Physicochemical Hydrodynamics: An Introduction, Butterworth Publishers, Stoneham 1988.

[41] Fuerstman, M. J., Lai, A., Thurlow, M. E., Shevkoplyas, S. S., Stone, H. A., Whitesides, G. M., Lab Chip 2007, 7, 1479-1489. 